(C) [2005] IEEE. Reprinted, with permission, from [Tang Zhongwei; Sanagavarapu Ananda, Effect of the strongest multipath component on indoor MIMO performance, Antennas and Propagation Society International Symposium, 2005 IEEE]. This material is posted here with permission of the IEEE. Such permission of the IEEE does not in any way imply IEEE endorsement of any of the University of Technology, Sydney's products or services. Internal or personal use of this material is permitted. However, permission to reprint/republish this material for advertising or promotional purposes or for creating new collective works for resale or redistribution must be obtained from the IEEE by writing to pubs-permissions@ieee.org. By choosing to view this document, you agree to all provisions of the copyright laws protecting it 


\title{
Effect of the Strongest Multpath Component on Indoor MIMO Performance
}

\author{
Zhongwei Tang* and Ananda S. Mohan \\ Microwave and Wireless Technology Research Lab, I\&C Group, \\ Faculty of Engineering, University of Technology, Sydney. \\ PO.Box 123, Broadway, Sydney, NSW 2007, Australia \\ zhongwei@eng.uts.edu.au, ananda@eng.uts.edu.au
}

\section{Introduction}

The multiple-input multiple-output (MIMO) technique $[1,2]$ has been drawing considerable attention recently, for its prospect for significant capacity improvement for wireless communications at no cost of extra spectrum. The motivation of MIMO systems is to achieve higher throughputs within a given bandwidth by using multiple antennas at both the transmitter and receiver to exploit the spatial dimension in addition to time and frequency dimensions.

A narrow band MIMO channel with $n_{t}$ transmit and $n_{r}$ receiver antennas is described in terms of a $n_{r} \times n_{t}$ complex channel transfer matrix $H$, whose entry $h_{i j}$ represents the normalized transfer function between the $i^{\text {th }}$ receive antenna to the $j^{\text {th }}$ transmit antenna. The generalized Shannon bound on channel capacity with uniform power allocation scheme is given by $[1,2]$ :

$$
C=\log _{2} \operatorname{det}\left(I+\frac{\rho}{n_{t}} H H^{*}\right)
$$

where $\rho$ is the average received signal to noise ratio (SNR).

Initial theoretical studies have assumed zero correlation between the antenna array elements, which would lead to an ideal channel matrix with independent and identically distributed (i.i.d) entries [1,2]. However, in reality, the practical channels are correlated [3] due to complex propagation features in both indoor and outdoor scenarios. Thus to understand and accurately describe or model indoor MIMO channels, channel measurements are the most straightforward way to distinguish the real propagation features of MIMO channels.

The effect of the line-of-sight (LOS) component in Ricean channels on the achievable MIMO capacity was theoretically investigated in $[4,5]$, where it was shown that the ergodic capacity decreases with the increase in the contribution of the LOS component. It is well known that the contribution of the strongest multipath component is represented by the Ricean $K$ factor. For outdoor Ricean MIMO channels, the effect of LOS component on capacity was investigated in [6]. However, experimental validation of the effect of LOS component in indoor Ricean MIMO channels is not reported so far in the open literature. In this paper, we experimentally investigate the effect of the strongest multipath component on indoor MIMO capacity in practical LOS and NLOS indoor environments. We show the indoor MIMO capacity increases with the decrease in the Ricean $K$ factor.

\section{Measurement Systems, Environments and Procedure}

The indoor MIMO channel measurements were performed inside LDC1 located on the $25^{\text {th }}$ floor of the 28-storey Tower Building of the University of Technology, Sydney (UTS). The schematic of the classroom is shown in Fig.1. The measurements were performed using a vector network analyzer (VNA) HP 8720A at a centre frequency of $2.45 \mathrm{GHz}$ for vertical polarization inside the classroom. The measurements were conducted during weekends to avoid the movement of people so as to approximate a quasistatic channel condition. Both the transmit and receive arrays were formed as synthetic arrays using commercially available sleeve dipole antennas in order to avoid mutual coupling and also to reduce the complexity and cost of the MIMO measurement. The return loss of the two sleeve dipole antennas was measured to be below $-15 \mathrm{~dB}$ within the 
considered bandwidth. To obtain a virtual transmit array, a computer controlled angular scanner moved a sleeve dipole antenna around a circle to form a virtual uniform circular array. At the receiver, the virtual uniform rectangular receive array was obtained using a computer controlled $\mathrm{X}-\mathrm{Y}$ scanning system to move a dipole antenna over the horizontal plane. For each transmitterreceiver configuration, 801 frequency response measurements were acquired within a bandwidth of $120 \mathrm{MHz}$.

The LDC1, as depicted in Fig.1, is a large open space room with an experimental-chamber, located at level 25 of the Tower Building. LDC1 has an open area with dimensions of $21.20 \times 9.50 \mathrm{~m}^{2}$. It has an external reinforced concrete wall on one side with a wide metalframed glass window and brick walls separating it from adjacent rooms of this level. Besides, a concrete wall separates it from stair cases. Within LDC1, a long counter table, $1.50 \mathrm{~m}$ above floor level, is located alongside the internal concrete wall. Cubicles, constructed with soft office-partitions with a height of $1.60 \mathrm{~m}$, are located along the two side walls, and set with tables and computers. The open area has wooden desks and plastic chairs for study and discussion. There is a rectangular-shaped experimental-chamber with a dimension of 5.40×7.40 $\mathrm{m}^{2}$ at one end of LDC1, with a single nest wooden door entrance. A thin brick wall separates the experimental-chamber from the open space of LDC1.

For the measurements in LDC1, referring to Fig.1, the transmit antenna was first fixed at a position in the experimental-chamber of LDC1 to perform NLOS channel measurements, and the receiver was successively located at different points as shown in the figure. The scenarios were under NLOS condition as the direct path between the transmit and receiver antennas was obstructed by the walls of the experimental-chamber. Later, in order to acquire LOS channel data, the transmit antenna was relocated to LC in LDC1 and the receiver was successively repositioned inside the open area of LDC1. Both the transmit and receive antennas were fixed at a height of $1.7 \mathrm{~m}$ above the floor in all measurements.

\section{Data Processing}

In the measurements, a 4-element synthetic uniform circular array with a radius of half a wavelength was formed at the transmitter and a 4-element synthetic planar square array with an interelement-spacing of half a wavelength was synthesized at the receiver. At each receiving position, a total of nine 4-element synthetic planar square arrays were formed. Therefore, for each transmitter-receiver pair, a total of $7209(9 \times 801)$ spatial and frequency $4 \times 4$ MIMO realizations were obtained.

To calculate the measured channel capacity, it is necessary that the acquired channel transfer matrices be normalized. We used the Frobenius norm in our calculations, given by:

$$
\sum_{i=1}^{n_{t}} \sum_{j=1}^{n_{r}}\left|h_{j i}\right|^{2}=n_{r} n_{t}
$$

where $h_{j i}$ is the element of the channel transfer matrix, $n_{t}$ and $n_{r}$ are the number of transmit and receive array elements.

The Ricean $K$ factor is defined as the ratio of the fixed to variable power components. We estimate the mean $K$ factor for each MIMO connection by using the moment-method [7] on the acquired data that are averaged over all the SISO channels for each position. The $K$ factor is calculated as:

$$
K=\frac{\sqrt{1-\gamma}}{1-\sqrt{1-\gamma}}
$$

where $\gamma=\sigma_{r}^{2} / P_{r}^{2}, \sigma_{r}$ is the variance of the receive signal power about its mean $P_{r}$.

\section{Measurement Results and Discussions}


The theoretical studies in $[4,5]$ on the effect of the line-of-sight component in Ricean channels on the achievable MIMO capacity showed that the increasing contribution of the LOS component degrade the ergodic capacity. This section, we experimentally study the effect of the Ricean $K$ factor on MIMO performance for both LOS and NLOS indoor scenarios. It is well known that the Ricean $K$ factor represents the effect of the strongest multipath component.

We have classified our measurements as LOS or NLOS data according to whether or not a direct line-of-sight path exists between the transmit and receive antennas. In our data, the strong direct component is always present even under NLOS condition. The only difference is that smaller value of $K$ factor results under NLOS channels as compared with LOS conditions. Fig. 2 presents the CDF plots of $K$ factor for measured LOS and NLOS MIMO channels. Fig.3 presents a comparison of CDFs of capacity between LOS, NLOS and i.i.d cases.

Figs. 4 and 5 show the scatter plots for the obtained MIMO capacity as a function of Ricean $K$ factor for LOS and NLOS channels. The obtained $4 \times 4$ MIMO capacity is calculated using equation (1) with SNR equal to $20 \mathrm{~dB}$. The MIMO channel is established between a 4-element transmit UCA and a 4-element square receive array. It shows that the reduction in LOS channel capacity with increasing $K$ factor. However, for NLOS scenarios, this trend is not as significant as that for LOS cases. The reason for this can be attributed to the rich multipath and smaller variation of $K$ factor, which ranges from 3-5 dB for NLOS scenario as compared to 3-7.8 dB for LOS channels. The effect of the strongest component on MIMO performance is minimized in NLOS cases due to rich multipath which results in smaller spatial correlation.

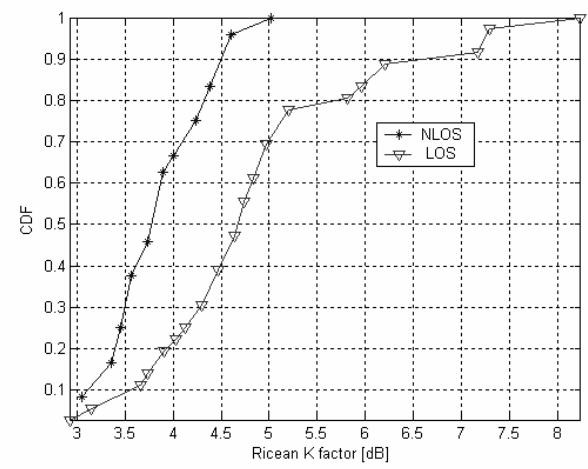

Fig.2. CDF of Ricean $K$ factor.

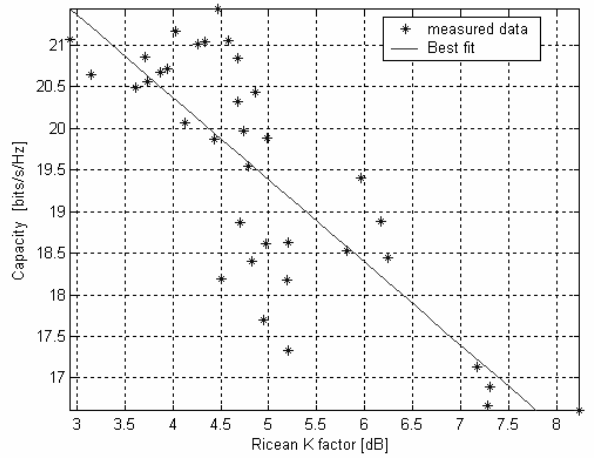

Fig.4. MIMO capacity versus Ricean $K$ factor for LOS channels.

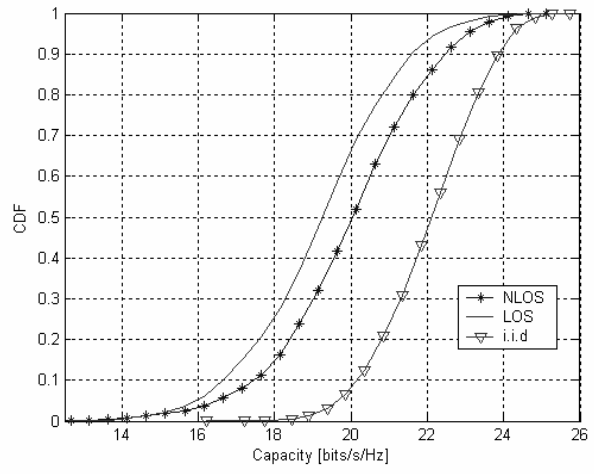

Fig.3. Comparison of MIMO capacity.

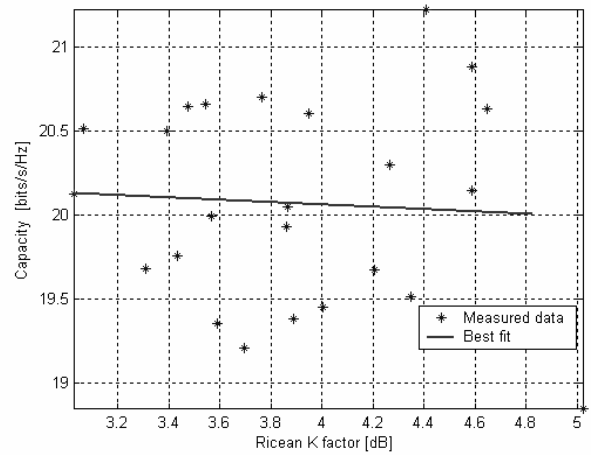

Fig.5. MIMO capacity versus Ricean $K$ factor for NLOS channels.

\section{Conclusions}

We have investigated the effect of the strongest multipath component on the achievable LOS/NLOS MIMO capacity in an indoor environment experimentally. Our results demonstrate 
the effect of the contribution of strongest component on indoor MIMO capacity. The LOS component is indeed detrimental for an indoor MIMO system to achieve high throughput due to it increase spatial correlation of the MIMO channel. In addition, the measured MIMO capacities are all less than that of the ideal uncorrelated i.i.d channels.

\section{Acknowledgement}

The project is funded by the Australian Research Council through a linkage grant program with Singtel Optus Pty Limited as the industry partner.

\section{References}

[1] I. E. Telatar, "Capacity of multi-antenna gaussian channels," Eueopean Trans. Telecommunications, vol. 10, pp. 585-595, Nov. 1999.

[2] G. J. Foschini and M. J. Gans, "On limits of wireless communications in a fading environment," Wireless Personal Communications, vol. 6, pp. 311-335, Mar.1998.

[3] K. Sulonen, P. Suvikunnas, L. Vuokko, J. Kivinen, and P. Vainikainen, "Comparison of MIMO antenna configurations in picocell and microcell environments," IEEE $J$. Select Areas Commun., vol. 21, pp. 703 - 712, June 2003.

[4] M. A. Khalighi, J.-M. Brossier, G. Jourdain, and K. Raoof, "On capacity of Rician MIMO channels," in proc. IEEE Int. Symp. on Personal, Indoor and Mobile Radio Communications, 2001, 2001, vol. 1, pp. A-150 -A-154.

[5] G. Lebrun, M. Faulkner, M. Shafi, and P. J. Smith, "MIMO Ricean Channel Capacity," in proc. 2004 IEEE Iternational conference on Comm., 2004, vol. 5, pp. 2939-2943.

[6] V. Erceg, P. Soma, D. S. Baum, and A. J. Paulraj, "Capacity obtained from multipleinput multiple-output channel measurements in fixed wireless environments at 2.5 GHz," in proc. IEEE International Conference on Communications, 2002, vol. 1, pp. $396-400$.

[7] L. J. Greenstein, D. G. Michelson, and V. Erceg, "Moment-method estimation of the Ricean K-factor," IEEE Comm. Lett., vol. 3, pp. 175-176, June 1999.

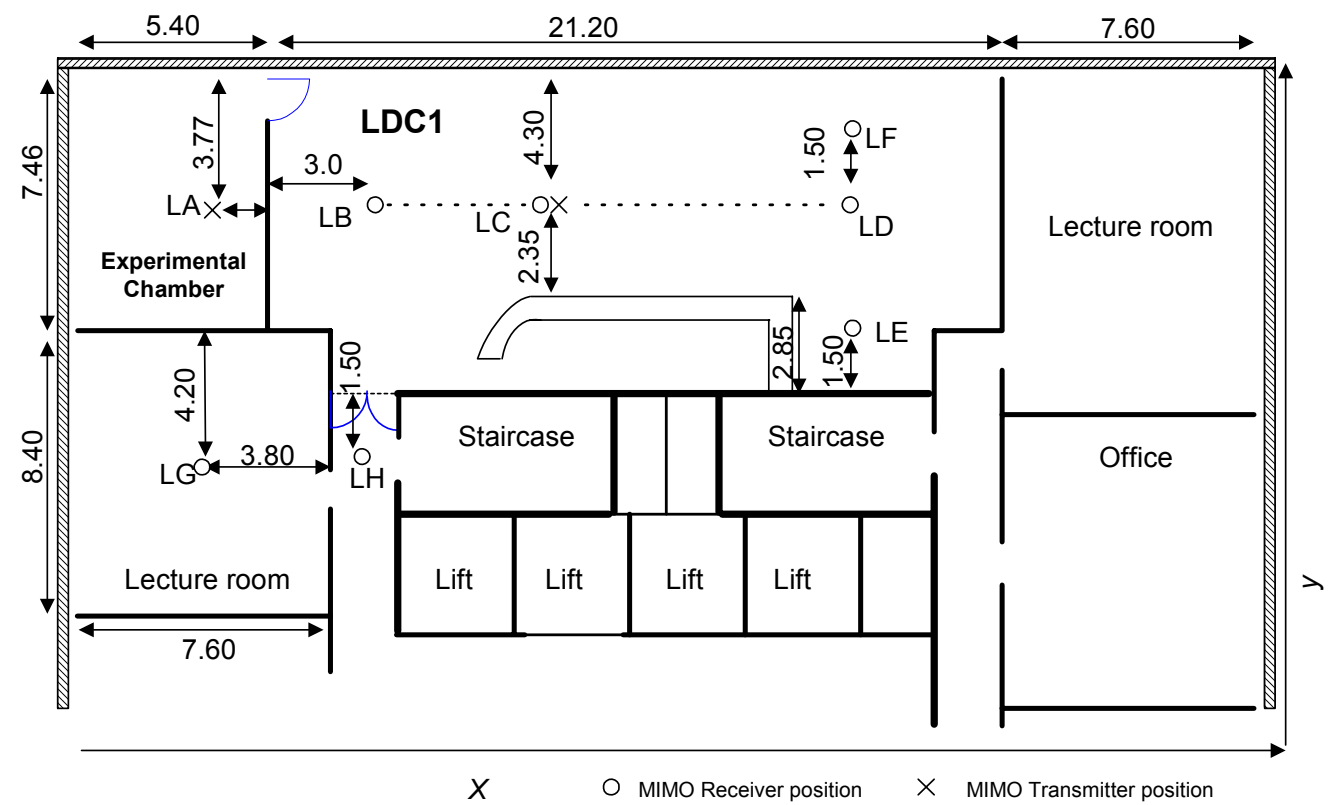

Fig.1. The plan of $25^{\text {th }}$ floor where LDC1 is located. 